\title{
Chlamydia trachomatis in sexually active teenage girls. Factors related to genital chlamydial infection: A prospective study
}

\author{
V A Rahm, V Odlind, R Pettersson
}

\begin{abstract}
The incidence of new infections with $C$ trachomatis was found to be $19 \%$. Predisposing factors for a subsequent chlamydial infection were multiple partners, smoking and previous infection with $C$ trachomatis. Girls with a spread cervical ectopy were not more likely to contract a chlamydial infection in one year than girls without an ectopy. Oral contraceptive use was not found to predispose for a chlamydial infection.
\end{abstract}

\section{Introduction}

Many reports in recent years have shown that Chlamydia trachomatis is frequently found in young sexually active women. ${ }^{1-5}$ Users of oral contraceptive pills (OC) have been reported to be at greater risk for genital infection with $C$ trachomatis in some studies, whereas others have failed to demonstrate such an association. Washington et $a l^{6}$ reviewed published data in order to clarify the relationship between OC use and chlamydial infection and reported a correlation, which was, however, less apparent in the adolescent population. Some reports indicate that steroid hormones enhance the growth, survival and ascension of genital chlamydial infection in animal models. ${ }^{78}$ However, Blum et $a l^{9}$ concluded that the risk of chlamydial infection seen in OC users may be derived from the sexual lifestyle rather than from a stimulative effect of the pill.

An association between a cervical ectopy and a high prevalence of chlamydial infection has often been reported. ${ }^{11011} \mathrm{We}$ have previously reported that friability of the cervix at sampling was associated with a chlamydial infection. ${ }^{12}$ However, in a study of the prevalence of chlamydial infection it is not possible to define whether a certain factor constitutes

Department of Obstetrics and Gynaecology, University of Uppsala, 75185 Uppsala, Sweden

V A Rahm

V Odlind

Department of Statistics, University of Uppsala, 751

20 Uppsala, Sweden

R Pettersson an increased risk for or is a consequence of the infection.

The aim of the present study was to investigate the incidence of new cases of $C$ trachomatis in one year and to characterise those adolescent girls most at risk.

\section{Material and methods}

The study population consisted of 301 sexually active teenage girls attending an adolescence clinic during a period of three months. The majority $(83.4 \%)$ of the girls were healthy and came to the clinic for family planning, and the rest had genital symptoms or had a male partner with urogenital symptoms. According to the results of the chlamydial testing at the initial visit, the girls were asked to return to the clinic at six and/or twelve months. A total of 268 girls were examined. The mean age of the girls at the initial visit was 17.7 years and the age ranges were between 14 and 19 years.

The girls were interviewed and information regarding current contraceptive method, current symptoms of genital infection as well as smoking habits and number of sexual partners during the last three months was obtained at every visit. At the twelve months follow-up visit the history also included the number of partners during the last year and the total (life-time) number of sexual partners.

A speculum examination was performed and the cervix was visualised and described at every visit. The extent of the ectopy was scored as no ectopy, ectopy involving less than one fourth (moderate) or ectopy involving one fourth or more of the visible ectocervix (spread). Specimens for chlamydial culture were obtained from each patient at every followup visit. Specimens were taken with an ENT swab after careful cleansing of the portio by a large dry cotton swab. Specimens were always obtained both from the endocervix and from the surface of the portio as previously reported. ${ }^{12}$

The swabs were immersed in tubes containing 2 SP medium and kept at $+4^{\circ} \mathrm{C}$ until they were transported to the laboratory within 24 hours of sampling. Cultures for $C$ trachomatis were made in cycloheximide treated McCoy cells. 
Table 1 The relation between results of chlamydial cultures and number of elementary bodies (EB) detected with direct fluorescein assay ( $D F A$ )

\begin{tabular}{lcc}
\hline$D F A$ & $\begin{array}{l}\text { Culture positive } \\
(n=53)\end{array}$ & $\begin{array}{l}\text { Culture negative } \\
(n=248)\end{array}$ \\
\hline None & 6 & 149 \\
$1-4$ & 19 & 95 \\
$5-9$ & 8 & 4 \\
$10-99$ & 15 & - \\
$<100$ & 5 & - \\
\hline
\end{tabular}

During the time of this study a non-culture test was also used. Specimens from the endocervix were smeared onto glass slides, fixed with acetone, transported and processed as a direct fluorescein assay (DFA) using FITC-conjugated monoclonal antibodies to $C$ trachomatis as previously described by Syva Company (Palo Alto, California). Elementary bodies (EB) were counted in every smear and a sample was scored as positive if at least $10 \mathrm{EBs}$ were visualised. For the follow-up the girls were divided into three groups according to the culture and DFA results. Group $1(n=53)$ consisted of those who were culture positive, group $2(n=99)$ of those who were culture negative but had smears with at least one EB. Group $3(n=149)$ consisted of girls who were both culture negative and had no EBs in DFA smears. The relation between culture and DFA results is seen in table 1 . Of those 95 slides where $1-4$ EBs were reported, the majority (81) had only one or two EBs. An immediate repeat test was performed in those girls who were culture negative but had more than four EBs in the DFA. They were and remained culture negative. Groups 1 and 2 were followed-up at 6 and 12 months, whereas group 3 was followed-up only at 12 months. No further analysis of the results from the DFA will be given here, but since this test constituted the reason for dividing the whole material into subgroups for follow-up, the procedure is reported.

All the chlamydia culture positive girls received treatment (lymecycline $300 \mathrm{mg} \times 2$ for ten days). No girl was chlamydia culture negative and DFA positive ( $>9 \mathrm{~EB})$. The same treatment was always given to the current sexual partner, whether he was chlamydia culture positive or not. Both were asked to abstain from sexual intercourse until the treatment was completed.

\section{Statistical analysis}

The data were recorded on precoded proformas and analysed with a computer using the Statistical Analysis System (SAS). Tests of significance were performed using the $\chi^{2}$ method with Yates's correction. A stepwise logistic regression analysis as well as loglinear models were used.
Table 2 The number of patients followed-up at 6 and 12 months and the number of chlamydia positive cultures in each group

\begin{tabular}{lcll}
\hline & $\begin{array}{l}\text { Initial } \\
\text { visit } n\end{array}$ & $\begin{array}{l}6 \text { months } \\
n \text { (Chlam pos) }\end{array}$ & $\begin{array}{l}12 \text { months } \\
n \text { (Chlam pos) }\end{array}$ \\
\hline Group 1 & 53 & $49(7)$ & $47(8)$ \\
Group 2 & 99 & $92(9)$ & $86(9)$ \\
Group 3 & 149 & - & $135(13)$ \\
\hline
\end{tabular}

\section{Results}

Chlamydia trachomatis was isolated from $53(17 \cdot 6 \%)$ of the 301 girls at the initial visit. All those who were positive and their current sexual partner claimed to have completed the given treatment. The follow-up at 6 and/or 12 months could therefore be considered a study of the coincidence of new infections with $C$ trachomatis.

A total of 268 girls were followed-up for 12 months. The number of patients followed-up in each group and the number of chlamydia positive cultures at the follow-up visits are seen in table 2. During the year of observation 46 of the girls became culture positive for $C$ trachomatis and another five had a chlamydial infection diagnosed and treated elsewhere, that is, an incidence of $19 \%$. Two girls (group 1) were chlamydia positive both at six and twelve months. They admitted having a new partner both at the six and twelve months visit. The incidence described as net cumulative probability per 100 woman-years was 26.8 in group $1,20.4$ in group 2 and $9 \cdot 6$ in group 3.

At the initial visit the majority of the girls $(82 \%)$ were current users of combined low dose OCs. Of the 268 girls followed-up, 179 had used OCs for at least 12 months, 35 girls started and 37 girls discontinued OC use during the study year and 17 girls had never used hormonal contraception.

Since previous studies have reported an association between use of OC, cervical ectopy and chlamydial infection, these factors were investigated prospectively. Girls who had used OCs for more than 12 months were more likely to have an ectopy than

Table 3 Change of the ectopy at 12 months as compared with the initial visit in relation to the duration of oral contraceptive use

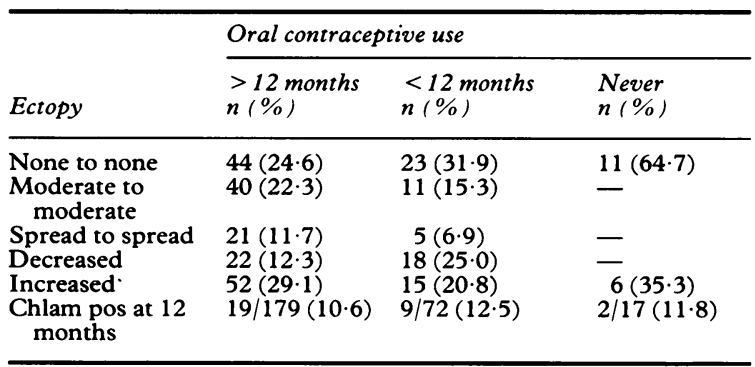


Table 4 Number of girls in the different groups at 6 and 12 months in relation to whether they had had the same partner throughout the study period or changed to a new partner or had different occasional sexual partners. Number of chlamydia positive girls is given within brackets

\begin{tabular}{|c|c|c|c|c|c|}
\hline & \multicolumn{2}{|l|}{6 months } & \multicolumn{3}{|c|}{12 months } \\
\hline & Group 1 & Group 2 & Group 1 & Group 2 & Group 3 \\
\hline $\begin{array}{l}\text { Current partner } \\
\text { New partner } \\
\text { Different partners }\end{array}$ & $\begin{array}{r}16(1) \\
4(1) \\
29(5)\end{array}$ & $\begin{array}{l}52(1) \\
10(1) \\
30(7)\end{array}$ & $\begin{array}{l}13 \\
11(3) \\
23(5)\end{array}$ & $\begin{array}{l}44(3) \\
17(1) \\
25(5)\end{array}$ & $\begin{array}{l}62(1) \\
29(4) \\
44(8)\end{array}$ \\
\hline
\end{tabular}

short-term and never users (table 3 ). When the cervical ectopy at the initial visit and that recorded after 12 months was compared in each individual, there was no statistically significant difference in the rate of increase of cervical ectopy between long-term and short-term OC users (table 3). Short-term users of OCs tended to exhibit a decrease of the size of the ectopy more often than those who had used OCs for more than 12 months ( $p<0.05$; table 3 ). Of those 40 girls whose ectopy decreased over the year, 12 had a chlamydial infection at the initial visit. All the five girls whose ectopy decreased from spread to none over the study year were chlamydia positive at the initial visit. They had all used OCs for more than 12 months.

Chlamydia culture positive girls reported significantly more partners the last year (mean 2.59 ) compared with culture negative girls (mean 1.79 ; $p<$ $0.01)$. Girls who claimed to have had the same partner throughout the study year were significantly less likely to have a new infection of $C$ trachomatis at 6 and/or 12 months than those who had changed to a new steady partner or admitted to have had different occasional partners (table 4). We were able to confirm that two of the male partners of those six chlamydia positive girls, who claimed to have had only one partner during the study period, had had a recent sexual relationship with another girl.

Factors that may predispose for $C$ trachomatis, such as number of partners, a spread ectopy, smoking and OC use, were compared in each of the three groups followed-up at twelve months. There was no difference in any of those factors between groups 2 and 3. Group 1, however, had a significantly larger mean number of life-time sexual partners compared

Table 5 Factors that may predispose for $C$ trachomatis in groups 1, 2, and 3 respectively

\begin{tabular}{llll}
\hline & $\begin{array}{l}\text { Group 1 } \\
(n=47)\end{array}$ & $\begin{array}{l}\text { Group 2 } \\
(n=86)\end{array}$ & $\begin{array}{l}\text { Group 3 } \\
(n=135)\end{array}$ \\
\hline $\begin{array}{l}\text { Mean number of life-time } \\
\text { partners }\end{array}$ & 7.4 & 4.3 & 4.4 \\
Spread ectopy (\%) & 25.5 & 18.6 & 16.3 \\
Smoking (\%) & 63.8 & 32.6 & 35.6 \\
OC-use (\%) & 78.7 & 81.4 & 79.3 \\
\hline Group 1 versus group 2 and 3: Life-time partners (p < 0.05),
\end{tabular}

spread ectopy (NS), smoking (p $<0.01$ ), OC use (NS). to groups 2 and 3. Smoking was twice as common in group 1 as in the other two groups $(p<0.001)$. There was, however, no statistically significant difference between the three groups with regard to a spread cervical ectopy, neither was there a difference in the rate of OC use (table 5).

The relative risk of a new chlamydial infection was significantly increased among girls who had a positive chlamydia culture at the first visit and among those who smoked (table 6). The relative risk for a new infection in the following year was slightly, but not significantly, increased in girls with a spread ectopy at their initial visit (table 6). Girls who, at their initial visit, had a friability at sampling and/or reported recent intermenstrual bleeding did not have an increased relative risk for a new infection during the subsequent year (table 6).

\section{Discussion}

This study has demonstrated an incidence of new cases of $C$ trachomatis of 19 per 100 woman-years among adolescent, sexually active girls, which corresponds to the prevalence found at the initial visit.

Those girls who had previously had an infection (group 1) were more likely to become reinfected during the study year which could be explained by the fact that this group reported more sexual partners, which underlines that $C$ trachomatis is a sexually transmitted disease. Other factors that appear as risk factors for genital infection, such as smoking in this study and OC use in some other studies, but not in the present one, could be considered interactive with sexual behaviour rather than causal. ${ }^{13-15}$

Table 6 The relative risk of chlamydial infection during a year of observation in relation to findings at the initial visit

\begin{tabular}{lll}
\hline & $R R$ & $\begin{array}{l}95 \% \text { confidence } \\
\text { interval }\end{array}$ \\
\hline Spread ectopy & $1 \cdot 78$ & $0.95-3.33$ \\
Smoking & $2 \cdot 21$ & $1 \cdot 28-3 \cdot 81$ \\
Chlamydia positive & 1.99 & $1 \cdot 12-3.54$ \\
Friability & 1.11 & $0.62-1.98$ \\
Intermenstrual bleed & 1.29 & $0.62-2 \cdot 69$ \\
Oral contraception & 0.67 & $0 \cdot 27-1.68$ \\
\hline
\end{tabular}


Oh et al concluded that OC use of 6 months or longer was associated with a risk of cervical chlamydial infection in teenage girls. ${ }^{14} \mathrm{OC}$ use did not predispose patients to contract chlamydial infection in our study population. This could be explained by the small group of $\mathrm{OC}$ non-users. However, girls having used OCs for more than one year were not more likely to have a chlamydial infection than those having used OCs for less than one year in the present study.

It could be argued that those who had an infection at their initial visit either did not comply completely with the treatment regimen or had a partner who did not comply and therefore continued to have a higher rate of infection throughout the observation year. All those who were given treatment were carefully instructed and all claimed to have understood the purpose of the treatment regimen and assured us that they had followed it strictly. No immediate posttreatment sample was obtained. We have previously performed a test of cure 1-2 weeks after completion of treatment in a population of 100 chlamydia culture positive teenage girls, who claimed to have taken their medication. No case of positive chlamydia culture was found, suggesting good compliance to given treatment, in this population. Other investigators have reported similar results of post-treatment chlamydial testing. ${ }^{16}$

Groups 2 and 3 were almost identical in terms of number of partners, finding of a cervical ectopy and smoking habits. Still, girls belonging to group 2 were twice as likely as those in group 3 to become infected in a year. One possible explanation for this difference could be that among those who were shown to have a reaction in the DFA, but were culture negative (group 2), some were false culture negative. However, out of the 99 girls belonging to group 2, only four had a DFA with more than four elementary bodies (EB) and none of those four girls became culture positive during the follow-up period. The most likely explanation for the difference between groups 2 and 3 is the fact that girls belonging to group 2 were examined more often, both at 6 and 12 months, whereas girls in group 3 only were sampled at 12 months. This apparent relation between a shorter sampling interval and a higher rate of positive cultures may suggest a spontaneous healing in some cases or transition to a latent stage where the infection cannot be detected. We have previously reported that, in a group of chlamydia culture positive untreated girls, around $20 \%$ developed symptoms of genital infection within 3 months, whereas $20 \%$ spontaneously became culture negative, when followed up. ${ }^{4}$

Many authors have reported a strong correlation between a cervical ectopy and chlamydial infection. ${ }^{10}{ }^{11} 17$ A cervical ectopy has also been reported to be more common in OC users than in women using non-hormonal contraception, possibly suggesting that $\mathrm{OC}$ use may to some extent contribute to the development of an ectopy. ${ }^{14}{ }^{15}$ Although an ectopy is a common finding in young girls, it is not clear to which extent low-dose OC use increases the incidence or the size of an ectopy in this age group. Since they probably differ in terms of number of sexual partners, frequency of intercourse and use of barrier methods for contraception, it will always be difficult to compare OC users with OC non-users for the hormonal effect on the ectopy and on infectivity of $C$ trachomatis. In our study population we found that girls who had used oral contraceptives for more than 12 months were more likely to have an ectopy than short-term and never users. A decreasing ectopy was more common in girls who had used oral contraceptives for less than 12 months. These findings may suggest that OC use does contribute to the development of an ectopy.

A spread ectopy at the initial visit did not clearly predispose for an infection during the subsequent year. A current infection could theoretically manifest itself as an ectopy and therefore appear as a risk factor in a prevalence study. Among those who reduced their ectopy from spread at the initial visit to none at the 12 month visit all had a chlamydial infection at their initial visit, suggesting that a spread ectopy may be a manifestation of a current infection.

This study has again confirmed that $C$ trachomatis is frequently found in adolescent, sexually active girls and that the infection in most cases is asymptomatic. $C$ trachomatis seems to be more related to life-style than to clinical signs. A new sexual partner, previous history of chlamydial infection and smoking were predisposing factors for contracting a chlamydial infection. The results clearly suggest that partner change is associated with a higher risk of new infection.

The study, showing an incidence of new cases of infection in a year of around $20 \%$, underlines the need for screening programmes for $C$ trachomatis in young sexually active individuals. They clearly constitute an important concealed reservoir for the infective agent and the only way of reducing this reservoir is to introduce routine screening for $C$ trachomatis, followed up with treatment and partner tracing.

We thank associate professor Håkan Gnarpe and his staff for expert handling of all the bacteriological specimens and for constant support. We also thank Mrs Gerd Rosen and the staff of the adolescent clinic, Gävle Ungdomsmottagning, for excellent cooperation during the course of the study.

Address correspondence to Dr V A Rahm. 
1 Chacko M, Lovchik J. Chlamydia trachomatis infection in sexually active adolescents: Prevalence and risk factors. Paediatrics 1984;73:836-40.

2 Hardy P, Nell E, Spence M, Hardy J, Graham D, Rosenbaum R. Prevalence of sexually transmitted disease agents among pregnant inner-city adolescents and pregnancy outcome. Lancet 1984;ii:333-7.

3 Handsfield H, Jasman L, Roberts P, Hanson V, Kothenbeutel R, Stamm W. Criteria for selective screening for Chlamydia trachomatis infection in women attending family planning clinics. JAMA 1986;255:1730-4.

4 Rahm VA, Gnarpe H, Odlind V. Chlamydia trachomatis among sexually active teenage girls. Lack of correlation between chlamydial infection, history of the patient and clinical signs of infection. Br J Obstet Gynaecol 1988;95:916-9.

5 Smith P, Phillips L, Faro S, McGill L, Wait R. Predominant sexually transmitted diseases among different age and ethnic groups of indigent sexually active adolescents attending a family planning clinic. J Adolesc Health Care 1988;9:291-5.

6 Washington AE, Gove S, Schachter J, Sweet R. Oral contraceptives, Chlamydia trachomatis infection and pelvic inflammatory disease: A word of caution about protection. JAMA 1985; 253:2246-50.

7 Rank R, White H, Hough A, Pasley J, Barron A. Effect of estradiol on chlamydial genital infection of female guineapigs. Infect Immun 1982;38:699-705.

8 Tuffrey $M$, Taylor-Robinson D. Progesteron as a key factor in the development of a mouse model for genital-tract infection with Chlamydia trachomatis. FEMS Microbiol Lett 1981; 121:111-5.

9 Blum M, Pery J, Kitai E. The link between contraceptive methods and Chlamydia trachomatis infection. Adv
Contracept 1988;4:233-9.

10 Harrison R, Costin M, Meder J, et al. Cervical Chlamydia trachomatis infection in university women: Relationship to history, contraception, ectopy, and cervicitis. Am J Obstet Gynecol 1985;153:244-51.

11 Blythe M, Katz B, Orr D, Caine V, Jones R. Historical and clinical factors associated with Chlamydia trachomatis genitourinary infection in female adolescents. $J$ Paediatr 1988;112:1000-4.

12 Rahm VA, Odlind V, Gnarpe H. Chlamydia trachomatis among sexually active teenage girls: influence of sampling location and clinical signs on the detection rate. Genitourin Med 1990; 66:66-9.

13 Wölner-Hanssen P, Eschenbach D, Paavonen J, et al. Decreased risk of symptomatic chlamydial pelvic inflammatory disease associated with oral contraceptive use. JAMA 1990;263:54-9.

14 Oh K, Feinstein R, Soileau E, Cloud G, Pass R. Chlamydia trachomatis, cervical infection and oral contraceptive use among adolescent girls. J Adolesc Health Care 1989;10: 376-81.

15 Louv W, Austin H, Perlman J, Alexander J. Oral contraceptive use and risk of chlamydial and gonococcal infections. Am J Obstet Gynecol 1989;160:396-401.

16 Radcliffe KW, Rowen D, Mercey DE, et al. Is a test of cure necessary for cervical infection with Chlamydial trachomatis? Genitourin Med 1990;66:444-6.

17 Kovacs G, Westcott M, Rusden J, et al. The prevalence of Chlamydia trachomatis in a young, sexually-active population. Med J Aust 1987;147:550-2.

Accepted for publication 6 May 1991 